PROCEEDINGS OF THE

AMERICAN MATHEMATICAL SOCIETY

Volume 137, Number 7, July 2009, Pages 2299-2309

S 0002-9939(09)09790-1

Article electronically published on March 3, 2009

\title{
A PAYNE-WEINBERGER EIGENVALUE ESTIMATE FOR WEDGE DOMAINS ON SPHERES
}

\author{
JESSE RATZKIN AND ANDREJS TREIBERGS \\ (Communicated by Chuu-Lian Terng)
}

\begin{abstract}
A Faber-Krahn type argument gives a sharp lower estimate for the first Dirichlet eigenvalue for subdomains of wedge domains in spheres, generalizing the inequality for the plane, found by Payne and Weinberger. An application is an alternative proof to the finiteness of a Brownian motion capture-time estimate.
\end{abstract}

Many lower estimates for the first Dirichlet eigenvalue of a domain stem from an inequality between a line integral and an area integral [Ch, pp. 85-133], LT, pp. 37-40], [P pp. 462-467]. These inequalities are often sharp, in that equality of the eigenvalues implies a geometric equality. For example, the Faber-Krahn inequality $[\mathrm{F},[\mathrm{K}$, proved by comparing level sets of the eigenfunction using the classical isoperimetric inequality, reduces to equality for round disks. Cheeger's inequality $[$ C bounds the eigenvalue from below in terms of the minimal ratio of area to length of subdomains.

Our main result, Theorem 1, is a lower bound for the first Dirichlet eigenvalue for a domain contained in a wedge in a two-sphere, generalizing an eigenvalue estimate of Payne and Weinberger [PW], $\mathrm{P}$, p. 462] for planar domains contained in a wedge. As an application, we give an alternative proof of our Brownian capturetime estimate $[\mathrm{RT}$. Curiously, our proof does not seem to carry over to domains contained in a wedge in the hyperbolic plane.

If $(\rho, \theta)$ are polar coordinates on $\mathbb{S}^{2}$, recall that the round metric is given by

$$
d s^{2}=d \rho^{2}+\sin ^{2} \rho d \theta^{2} .
$$

Let $\mathcal{W}=\{(\rho, \theta): 0 \leq \theta \leq \pi / \alpha, 0 \leq \rho<\pi\}$ be the sector in $\mathbb{S}^{2}$ of angle $\pi / \alpha$, for $\alpha>1$, and let $G$ be a domain such that $\bar{G} \subset \mathcal{W}$ is compact. Also define the truncated sector $\mathcal{S}(r):=\{(\rho, \theta): 0 \leq \theta \leq \pi / \alpha, 0 \leq \rho \leq r\}$. Observe that

$$
w=\tan ^{\alpha}\left(\frac{\rho}{2}\right) \sin \alpha \theta
$$

is a positive harmonic function in $\mathcal{W}$, with zero boundary values.

Theorem 1. For every subdomain $G$ with compact $\bar{G} \subset \mathcal{W}$, we have the estimate

$$
\lambda_{1}(G) \geq \lambda_{1}\left(\mathcal{S}\left(r^{*}\right)\right)
$$

Received by the editors April 10, 2008.

2000 Mathematics Subject Classification. Primary 35P15.

(C)2009 American Mathematical Society Reverts to public domain 28 years from publication 
where $r^{*}$ is chosen such that

$$
\mathcal{I}(G)=\int_{G} w^{2} d a=\int_{\mathcal{S}\left(r^{*}\right)} w^{2} d a .
$$

Equality holds if and only if $G$ is the sector $\mathcal{S}\left(r^{*}\right)$ almost everywhere.

Our argument is similar to the proof of the planar version in $[\mathrm{PW}$. Our main tool is an isoperimetric-type inequality, Lemma 3, which we prove in Section 1. We use this inequality to estimate the Rayleigh quotient of a test function, proving Theorem 11 in Section 2. Finally, in Section 3, we apply our eigenvalue estimate to a problem in Brownian pursuit.

\section{ISOPERIMETRIC INEQUALITY}

In this section we prove an isoperimetric inequality for moments of inertia of a domain $G \subset \mathcal{W}$. Later we will use this inequality to estimate the Raleigh quotient of admissible functions in $G$.

We begin by stating a version of Szegö's Lemma $[\mathrm{Sz}$ :

Lemma 2. Let $\psi, \phi:[0, \omega) \rightarrow[0, \infty)$ be locally integrable functions with $\psi$ nonnegative and $\phi$ nondecreasing. Let $\Phi(y)=\int_{0}^{y} \phi(t) d t$ and $\Psi(x)=\int_{0}^{y} \psi(s) d s$ be their primitives. Let $E \subset[0, \omega)$ be a bounded measurable set. Then

$$
\Phi\left(\int_{E} \psi(x) d x\right) \leq \int_{E} \phi(\Psi(x)) \psi(x) d x .
$$

For $\phi$ increasing, equality holds if and only if the measure of $E \cap[0, R]$ is $R$.

Proof. Let $\mu$ be the Lesbesgue measure with line element $d x$ and define the measure $\nu$ by $d \nu=\psi d x$. Then $\nu$ is absolutely continuous with respect to $\mu$ and, using the Radon-Nikodym Theorem, when we change variables $y=\Psi(x)$ we have $d y=$ $\psi(x) d x$. Let $E^{\prime}$ be the image of $E$ under the map $\Psi$, with characteristic function $\chi_{E^{\prime}}$, so that $\Phi\left(\int_{E^{\prime}} d y\right)=\Phi\left(\int_{E} \psi(x) d x\right)$. Next, because $\phi$ is nondecreasing, for $y \geq 0$,

$$
\phi\left(\int_{0}^{y} \chi_{E^{\prime}} d y\right) \leq \phi(y) .
$$

Moreover, for $\phi$ increasing, equality holds if and only if $\mu\left(E^{\prime} \cap[0, y]\right)=y$. We multiply this inequality by $\chi_{E^{\prime}}$ and integrate:

$$
\int_{0}^{\omega} \phi\left(\int_{0}^{y} \chi_{E^{\prime}} d t\right) \chi_{E^{\prime}} d y \leq \int_{0}^{\omega} \phi(y) \chi_{E^{\prime}} d y=\int_{E^{\prime}} \phi(y) d y=\int_{E} \phi(\Psi(x)) \psi(x) d x .
$$

On the other hand,

$$
\int_{0}^{\omega} \phi\left(\int_{0}^{y} \chi_{E^{\prime}} d t\right) \chi_{E^{\prime}} d y=\Phi\left(\int_{E^{\prime}} d y\right)=\Phi\left(\int_{E} \psi(x) d x\right) .
$$

Putting these two inequalities together yields the inequality (3).

Lemma 3. Let $G \subset \mathcal{W}$ be a domain with compact closure. Then there is a function $\Upsilon_{\alpha}=\mathcal{F} \circ Z^{-1}$ so that

$$
\int_{\partial G} w^{2} d s \geq \frac{\pi}{2 \alpha} \Upsilon_{\alpha}\left(\frac{2 \alpha}{\pi} \int_{G} w^{2} d a\right) .
$$

Here $\mathcal{F}(\rho)=\tan ^{2 \alpha}(\rho / 2) \sin \rho$ and $Z$ is given by (11). Equality holds if and only if $G$ is a sector $\mathcal{S}(r)$ almost everywhere. 
Proof. Map the domain $G$ into a domain $\tilde{G}$ in the upper half-plane using the transformation

$$
x=f(\rho) \cos \alpha \theta, \quad y=f(\rho) \sin \alpha \theta,
$$

where we will choose $f$ to satisfy formula (8). The Euclidean line element is

$$
d x^{2}+d y^{2}=\dot{f}^{2} d \rho^{2}+\alpha^{2} f^{2} d \theta^{2} .
$$

We claim that the map satisfies

$$
\alpha^{2} \tan ^{4 \alpha}\left(\frac{\rho}{2}\right) \sin ^{4} \alpha \theta\left(d \rho^{2}+\sin ^{2} \rho d \theta^{2}\right) \geq y^{4}\left(d x^{2}+d y^{2}\right) .
$$

For this to be true pointwise, we need the following inequalities to hold:

$$
\begin{aligned}
\alpha \tan ^{2 \alpha}\left(\frac{\rho}{2}\right) & \geq f^{2} \dot{f}=\left(\frac{f^{3}}{3}\right)^{\prime}, \\
\sin \rho \tan ^{2 \alpha}\left(\frac{\rho}{2}\right) & \geq f^{3} .
\end{aligned}
$$

Expand $\sin \rho=2 \sin (\rho / 2) \cos (\rho / 2)$ and use equality in inequality (7) to define $f$ :

$$
f=2^{\frac{1}{3}} \sin ^{\frac{1+2 \alpha}{3}}\left(\frac{\rho}{2}\right) \cos ^{\frac{1-2 \alpha}{3}}\left(\frac{\rho}{2}\right) .
$$

Differentiating, we see that

$$
f^{2} \dot{f}=\tan ^{2 \alpha}\left(\frac{\rho}{2}\right)\left[\frac{2 \alpha+\cos \rho}{3}\right],
$$

which implies that the inequality (6) holds as well.

Equation (10) and inequality (5) imply that

$$
\alpha \int_{\partial G} w^{2} d s=\alpha \int_{\partial G} w^{2} \sqrt{d \rho^{2}+\sin ^{2} \rho d \theta^{2}} \geq \int_{\partial \tilde{G}} y^{2} \sqrt{d x^{2}+d y^{2}}:=\mathcal{M}(\partial \tilde{G}) .
$$

The right side is the moment of inertia of a uniform mass distribution of the curve $\partial \tilde{G}$ relative to the $y$-axis. Among all domains with given fixed surface moment

$$
\int_{\tilde{G}} y^{2} d x d y
$$

the semicircular arcs centered on the $y$-axis minimize $\mathcal{M}(\partial \tilde{G})$ [PW, Section 2]. Compute $\mathcal{M}(\partial \tilde{G})$ and $\mathcal{M}(\tilde{G})$ in the case where $\partial \tilde{G}$ is a semicircle of radius $R$ :

$$
\mathcal{M}(\partial \tilde{G})=\int_{0}^{\pi} R^{3} \sin ^{2} t d t=\frac{\pi R^{3}}{2}, \quad \mathcal{M}(\tilde{G})=\int_{0}^{\pi} \int_{0}^{R} r^{3} \sin ^{2} \theta d r d \theta=\frac{\pi R^{4}}{8} .
$$

Solving for $R$ in the formula for $\mathcal{M}(\tilde{G})$ above and using the fact that semicircles are minimizers, we see that for a general domain $\tilde{G}$ in the upper half-plane,

$$
\mathcal{M}(\partial \tilde{G}) \geq 2^{\frac{5}{4}} \pi^{\frac{1}{4}}\left\{\int_{\tilde{G}} y^{2} d x d y\right\}^{\frac{3}{4}} .
$$


Returning to the original variables, $d x d y=\alpha f \dot{f} d \rho d \theta$, so

$$
\begin{aligned}
& \int_{\partial G} w^{2} d s \geq \frac{1}{\alpha} 2^{\frac{5}{4}} \pi^{\frac{1}{4}}\left\{\int_{G} f^{2} \sin ^{2}(\alpha \theta) \alpha f \dot{f} d \rho d \theta\right\}^{\frac{3}{4}} \\
&=\left(\frac{\pi}{2 \alpha}\right)^{\frac{1}{4}}\left\{\int_{G} \frac{4}{3}\left[\tan ^{2 \alpha}\left(\frac{\rho}{2}\right) \sin \rho\right]^{\frac{1}{3}}\right. \\
& {\left.[2 \alpha+\cos \rho] \tan ^{2 \alpha}\left(\frac{\rho}{2}\right) \sin ^{2} \alpha \theta d \rho d \theta\right\}^{\frac{3}{4}} . }
\end{aligned}
$$

Choose $\beta$ so that

$$
\frac{2 \alpha+2}{2 \alpha+1} \leq \beta<\frac{4}{3}
$$

which is possible because $\alpha>1$. Regroup the integral inside the braces:

$$
\begin{aligned}
I=\frac{4}{3 \beta} \int_{G}[ & \left.\tan ^{2 \alpha}\left(\frac{\rho}{2}\right) \sin \rho\right]^{\frac{4}{3}-\beta} \\
& \times[2 \alpha+\cos \rho] \beta\left[\tan ^{2 \alpha}\left(\frac{\rho}{2}\right) \sin \rho\right]^{\beta-1} \tan ^{2 \alpha}\left(\frac{\rho}{2}\right) d \rho \sin ^{2} \alpha \theta d \theta .
\end{aligned}
$$

Use Lemma 2, with

$$
\Psi=\left[\tan ^{2 \alpha}\left(\frac{\rho}{2}\right) \sin \rho\right]^{\beta} \Rightarrow \psi=\beta\left(\tan ^{2 \alpha}\left(\frac{\rho}{2}\right) \sin \rho\right)^{\beta-1}[2 \alpha+\cos \rho] \tan ^{2 \alpha}\left(\frac{\rho}{2}\right)
$$

and

$$
\phi(z)=\frac{4}{3 \beta} z^{\frac{4}{3 \beta}-1} \Rightarrow \Phi(z)=z^{\frac{4}{3 \beta}} .
$$

So that $\phi$ is increasing, we require $\beta<\frac{4}{3}$. If $H_{\theta}=\{\rho \in[0, \pi):(\rho, \theta) \in G\}$ is the slice of $G$ in the $\rho$-direction, then Szegö's inequality (3) implies

$$
I \geq \int_{0}^{\pi / \alpha}\left(\beta \int_{H_{\theta}} \tan ^{2 \alpha \beta}\left(\frac{\rho}{2}\right) \sin ^{\beta-1} \rho[2 \alpha+\cos \rho] d \rho\right)^{\frac{4}{3 \beta}} \sin ^{2} \alpha \theta d \theta .
$$

Equality holds if and only if $H_{\theta}=[0, r(\theta)]$ is an interval a.e. Next we let $p=\frac{4}{3 \beta}>1$, $q=\frac{4}{4-3 \beta}$, and define the measure $d \nu=\sin ^{2} \alpha \theta d \theta$. Hölder's inequality implies

$$
\begin{gathered}
{\left[\int_{0}^{\pi / \alpha}\left(\beta \int_{H_{\theta}} \tan ^{2 \alpha \beta}\left(\frac{\rho}{2}\right) \sin ^{\beta-1}(\rho)[2 \alpha+\cos \rho] d \rho\right)^{p} d \nu\right]^{\frac{1}{p}}\left[\int_{0}^{\pi / \alpha} d \nu\right]^{\frac{1}{q}}} \\
\geq \int_{0}^{\pi / \alpha} \beta \int_{H_{\theta}} \tan ^{2 \alpha \beta}\left(\frac{\rho}{2}\right) \sin ^{\beta-1}(\rho)[2 \alpha+\cos \rho] d \rho d \nu .
\end{gathered}
$$

Raising both sides of this inequality to the power $p$, rearranging, and using the fact that

$$
\int_{0}^{\pi / \alpha} d \nu=\int_{0}^{\pi / \alpha} \sin ^{2} \alpha \theta d \theta=\frac{\pi}{2 \alpha}
$$

(10) becomes

$$
I \geq\left(\frac{2 \alpha}{\pi}\right)^{\frac{4}{3 \beta}-1}\left(\beta \int_{0}^{\pi / \alpha} \int_{H_{\theta}} \tan ^{2 \alpha \beta}\left(\frac{\rho}{2}\right) \sin ^{\beta-1} \rho[2 \alpha+\cos \rho] d \rho \sin ^{2} \alpha \theta d \theta\right)^{\frac{4}{3 \beta}} .
$$


We regroup the inside integral again:

$$
J=\int_{0}^{\pi / \alpha} \int_{H_{\theta}} \tan ^{2 \alpha(\beta-1)}\left(\frac{\rho}{2}\right) \sin ^{\beta-2} \rho[2 \alpha+\cos \rho] \tan ^{2 \alpha}\left(\frac{\rho}{2}\right) \sin \rho d \rho \sin ^{2} \alpha \theta d \theta .
$$

Let us denote

$$
Z(r)=\int_{0}^{r} \tan ^{2 \alpha}\left(\frac{\rho}{2}\right) \sin \rho d \rho
$$

and define $\bar{r}(r, \theta)$ by

$$
Z(\bar{r})=\int_{0}^{r} \tan ^{2 \alpha}\left(\frac{\rho}{2}\right) \chi_{H_{\theta}}(\rho) \sin \rho d \rho,
$$

where $\chi_{H}$ denotes the characteristic function of $H$. The integrand $\tan ^{2 \alpha}(\rho / 2) \sin \rho$ is positive and increasing for the range of $\rho$ we are considering, and so $\bar{r}(r, \theta) \leq r$ with equality if and only if $H_{\theta} \cap[0, r]=[0, r]$ a.e. If we require that $(2 \alpha+1) \beta \geq 2 \alpha+2$, then the factor

$$
g_{\beta}(\rho)=\tan ^{2 \alpha(\beta-1)}\left(\frac{\rho}{2}\right) \sin ^{\beta-2} \rho[2 \alpha+\cos \rho]
$$

is increasing in $\rho$. Thus we can define $\Phi_{\beta}$ by

$$
\phi_{\beta}(y)=\beta g_{\beta} \circ Z^{-1}(y), \quad \Phi_{\beta}(y)=\int_{0}^{y} \phi_{\beta}(s) d s .
$$

Observe that $Z$ and $g_{\beta}$ are increasing, so $\phi_{\beta}$ is increasing and $\Phi_{\beta}$ is convex. Using $g_{\beta}(\bar{r}(\rho, \theta)) \leq g_{\beta}(\rho)$, we have

$$
\begin{aligned}
J & \geq \int_{0}^{\pi / \alpha} \int_{H_{\theta}} g_{\beta}(\bar{r}(\rho, \theta)) \tan ^{2 \alpha}\left(\frac{\rho}{2}\right) \sin \rho d \rho \sin ^{2} \alpha \theta d \theta \\
& =\frac{1}{\beta} \int_{0}^{\pi / \alpha} \int_{H_{\theta}} \phi_{\beta}\left(\int_{0}^{\rho} \tan ^{2 \alpha}\left(\frac{\rho^{\prime}}{2}\right) \chi_{H_{\theta}}\left(\rho^{\prime}\right) \sin \rho^{\prime} d \rho^{\prime}\right) \tan ^{2 \alpha}\left(\frac{\rho}{2}\right) \sin \rho d \rho \sin ^{2} \alpha \theta d \theta .
\end{aligned}
$$

Now, using Lemma 2 with $\psi(\rho)=\tan ^{2 \alpha}(\rho / 2) \sin (\rho) \chi_{H_{\theta}}$ and $\phi_{\beta}$ given by equation (12), we have

$$
J \geq \frac{1}{\beta} \int_{0}^{\pi / \alpha} \Phi_{\beta}\left(\int_{H_{\theta}} \tan ^{2 \alpha}\left(\frac{\rho}{2}\right) \sin \rho d \rho\right) \sin ^{2} \alpha \theta d \theta
$$

with equality if and only if $H_{\theta}=[0, r(\theta)]$ is an interval a.e. Next, by Jensen's inequality (with the measure given by $d \nu=\sin ^{2} \alpha \theta d \theta$ ),

$$
J \geq \frac{\pi}{2 \alpha \beta} \Phi_{\beta}\left(\frac{2 \alpha}{\pi} \int_{0}^{\pi / \alpha} \int_{H_{\theta}} \tan ^{2 \alpha}\left(\frac{\rho}{2}\right) \sin ^{2} \alpha \theta \sin \rho d \rho d \theta\right)
$$

with equality if and only if $\bar{r}(\theta)$ is a.e. constant. Substituting back, we get

$$
\begin{aligned}
I & \geq\left(\frac{2 \alpha}{\pi}\right)^{\frac{4}{3 \beta}-1}(\beta J)^{\frac{4}{3 \beta}} \\
& \geq \frac{\pi}{2 \alpha}\left\{\Phi_{\beta}\left(\frac{2 \alpha}{\pi} \int_{0}^{\pi / \alpha} \int_{H_{\theta}} \tan ^{2 \alpha}\left(\frac{\rho}{2}\right) \sin ^{2} \alpha \theta \sin \rho d \rho d \theta\right)\right\}^{\frac{4}{3 \beta}} .
\end{aligned}
$$


Reinserting this back into (9) yields

$$
\begin{aligned}
\int_{\partial G} w^{2} d s & \geq\left(\frac{\pi}{2 \alpha}\right)^{\frac{1}{4}} I^{\frac{3}{4}} \geq \frac{\pi}{2 \alpha} \Phi_{\beta}^{\frac{1}{\beta}}\left(\frac{2 \alpha}{\pi} \int_{0}^{\pi / \alpha} \int_{H_{\theta}} \tan ^{2 \alpha}\left(\frac{\rho}{2}\right) \sin ^{2} \alpha \theta \sin \rho d \rho d \theta\right) \\
& =\frac{\pi}{2 \alpha} \Phi_{\beta}^{\frac{1}{\beta}}\left(\frac{2 \alpha}{\pi} \int_{G} w^{2} d a\right),
\end{aligned}
$$

where equality holds if and only if also $\rho(\theta)$ is constant a.e. Notice that the righthand side of this inequality is always bounded by $\int_{\partial G} w^{2} d s$, and so we can use the Dominated Convergence Theorem to take a limit as $\beta \rightarrow \frac{4}{3}$ from below. In other words, (13) also holds for $\beta=\frac{4}{3}$.

Let us compute $\Phi_{\beta}^{\frac{1}{\beta}}(Y)$. Since it depends only on (12), it would be the same for any function $v^{*}$ whose level sets $G_{\eta}^{*}=\left\{x: v^{*}(x) \geq \eta\right\}$ give the same value for the integral of $w^{2}$ (see (16) below). In this case, we choose a spherical rearrangement whose levels are the sectors $G_{\eta}^{*}=\mathcal{S}(r(\eta))$. Expressing things in terms of $r(\eta)$, we have

$$
\frac{2 \alpha}{\pi} y=\frac{2 \alpha}{\pi} \zeta(\eta)=\frac{2 \alpha}{\pi} \int_{\mathcal{S}(r(\eta))} w^{2} d a=Z(r(\eta))
$$

so, changing variables $s=Z(r)$ yields

$$
\begin{aligned}
\Phi_{\beta}(Y) & =\int_{0}^{Y} \phi_{\beta}(s) d s \\
& =\beta \int_{0}^{Z^{-1}(Y)} g_{\beta}(r) \tan ^{2 \alpha}\left(\frac{r}{2}\right) \sin r d r \\
& =\beta \int_{0}^{Z^{-1}(Y)}\left[\tan ^{2 \alpha}\left(\frac{r}{2}\right) \sin r\right]^{\beta-1}[2 \alpha+\cos r] \tan ^{2 \alpha}\left(\frac{r}{2}\right) d r \\
& =\left[\tan ^{2 \alpha}\left(\frac{Z^{-1}(Y)}{2}\right) \sin \left(Z^{-1}(Y)\right)\right]^{\beta} .
\end{aligned}
$$

Observe that we get the same equation (13) for all $\beta$. Thus we set $\Upsilon_{\alpha}=\Phi_{\beta}^{\frac{1}{\beta}}$ in (13), giving (4).

It is precisely at inequality (6) where the analogous proof in the hyperbolic case fails. In the hyperbolic case, the harmonic weight function is $w(\rho, \theta)=$ $\tanh ^{2 \alpha}(\rho / 2) \sin (\alpha \theta)$, and versions of equations (5), (8) hold with cos replaced by cosh and sin replaced by sinh. This choice of $f$ gives us

$$
f^{2} \dot{f}=\tanh ^{2 \alpha}\left(\frac{\rho}{2}\right)\left[\frac{2 \alpha+\cosh \rho}{3}\right],
$$

much like the formula above, but this does not yield $f^{2} \dot{f} \leq \alpha \tanh ^{2 \alpha}(\rho / 2)$, because $\cosh \rho$ grows exponentially with $\rho$. To remedy this problem, one can try to vary the power of $\sinh (\rho / 2)$ or $\cosh (\rho / 2)$; however this will only yield a worse inequality for $f^{2} \dot{f}$. 


\section{Estimate of RAYLeigh QUOTIENT}

Theorem 1 now follows as in [PW]. Let $G \subset \mathbb{S}^{2}$ be a domain that lies in the wedge $\mathcal{W}=\{(\rho, \theta): 0 \leq \rho, 0 \leq \theta \leq \pi / \alpha\}$. It suffices to estimate the Rayleigh quotient for admissible functions $u \in C_{0}^{2}(G)$ that are twice continuously differentiable and compactly supported in $G$. Any admissible function may be written $u=v w$ using the harmonic function (1) and $v \in C_{0}^{2}(G)$. The divergence theorem shows that

$$
\int_{G}|d u|^{2} d a=\int_{G} w^{2}|d v|^{2} d a .
$$

Let $G_{t}$ denote the points of $G$ satisfying $v \geq t$. Putting

$$
\zeta(t)=\int_{G_{t}} w^{2} d a,
$$

we see that $\zeta(0)=\hat{\zeta} \geq \zeta(t) \geq 0=\zeta(\hat{v})$, where $\hat{v}=\max _{G} v$,

$$
\frac{\partial \zeta}{\partial t}=-\int_{\partial G_{t}} \frac{w^{2}}{|d v|} d s
$$

and

$$
\int_{G} w^{2} v^{2} d a=\int_{0}^{\hat{v}} 2 t \zeta(t) d t=\int_{0}^{\hat{\zeta}} t^{2} d \zeta
$$

Then, using the coarea formula, Schwarz's inequality, Lemma 3 and changing variables to $y=\zeta(t)$, we find that inequality (4) implies

$$
\begin{aligned}
\int_{G} w^{2}|d v|^{2} d a & \geq \int_{0}^{\hat{v}}\left\{\int_{\partial G_{t}} w^{2}|d v| d s\right\} d t \\
& \geq \int_{0}^{\hat{v}} \frac{\left\{\int_{\partial G_{t}} w^{2} d s\right\}^{2}}{\int_{\partial G_{t}} \frac{w^{2}}{|d v|} d s} d t \\
& \geq \frac{\pi^{2}}{4 \alpha^{2}} \int_{0}^{\hat{v}} \frac{\Upsilon_{\alpha}^{2}\left(\frac{2 \alpha}{\pi} \zeta(t)\right)}{-\frac{\partial \zeta}{\partial t}} d t .
\end{aligned}
$$

Changing variables to $y=\zeta(t)$ we have

$$
\int_{0}^{\hat{\zeta}} \Upsilon_{\alpha}^{2}\left(\frac{2 \alpha}{\pi} y\right)\left(\frac{\partial t}{\partial y}\right)^{2} d y \geq \mu \int_{0}^{\hat{\zeta}} t(y)^{2} d y
$$

where $\mu$ is the least eigenvalue of the boundary value problem

$$
\begin{gathered}
\frac{\partial}{\partial y}\left(\Upsilon_{\alpha}^{2}\left(\frac{2 \alpha}{\pi} y\right) \frac{\partial q}{\partial y}\right)+\mu q=0, \\
q(\hat{\zeta})=0, \quad \lim _{y \rightarrow 0+} \Upsilon_{\alpha}^{2}\left(\frac{2 \alpha}{\pi} y\right) \frac{\partial q}{\partial y}=0 .
\end{gathered}
$$


Now perform the change of variables in (19) and (20) given by (14) so that the domain is now $\left[0, r^{*}\right], Z\left(r^{*}\right)=\frac{2 \alpha}{\pi} \hat{\zeta}$, and $\mu$ is now the least eigenvalue of

$$
\begin{gathered}
\frac{\partial}{\partial r}\left(\tan ^{2 \alpha}\left(\frac{r}{2}\right) \sin (r) \frac{\partial q}{\partial r}\right)+\frac{\pi^{2} \mu}{4 \alpha^{2}} \tan ^{2 \alpha}\left(\frac{r}{2}\right) \sin (r) q=0, \\
q\left(r^{*}\right)=0, \quad \lim _{r \rightarrow 0+} \tan ^{2 \alpha}\left(\frac{r}{2}\right) \sin (r) \frac{\partial q}{\partial r}=0 .
\end{gathered}
$$

Note that (21) is the eigenequation for the spherical sector $\mathcal{S}\left(r^{*}\right)$. Hence $\frac{\pi^{2} \mu}{4 \alpha^{2}}=$ $\lambda_{1}\left(\mathcal{S}\left(r^{*}\right)\right)$.

Reassembling, using equations (17) and (18), we get the inequality

$$
\int_{G}|d u|^{2} d a \geq \lambda_{1}\left(\mathcal{S}\left(r^{*}\right)\right) \int_{G} u^{2} d a
$$

which implies the inequality (2).

\section{Computation of the LOWER BOUnd AND APPLiCATions}

The eigenvalue $\lambda^{*}=\lambda_{1}\left(\mathcal{S}\left(r^{*}\right)\right)$ occurs as the eigenvalue of the problem (21), (22) on $\left[0, r^{*}\right]$, which may be rewritten as

$$
\begin{gathered}
\sin (r) q^{\prime \prime}+[2 \alpha+\cos (r)] q^{\prime}+\lambda^{*} \sin (r) q=0 ; \\
\lim _{r \rightarrow 0-} \tan ^{2 \alpha}\left(\frac{r}{2}\right) \sin (r) \frac{d q}{d r}(r)=0, \quad q\left(r^{*}\right)=0 .
\end{gathered}
$$

Making the change of variable $x=\frac{1}{2}(1-\cos r)$ transforms the ODE to the hypergeometric equation on $[0,1]$ :

$$
\begin{array}{cc}
x(1-x) \ddot{y}+[c-(a+b+1) x] \dot{y}-a b y & =0, \\
\lim _{x \rightarrow 0-} x^{\alpha+1} \frac{d y}{d r}(x)=0, & q\left(x^{*}\right)=0,
\end{array}
$$

with

$$
a, b=\frac{1 \pm \sqrt{1+4 \lambda^{*}}}{2}, \quad c=\alpha+1
$$

The solution to the hypergeometric equation is Gauß's ordinary hypergeometric function, given by

$$
\begin{aligned}
{ }_{2} \mathrm{~F}_{1}(a, b ; c ; x)= & 1+\frac{a b}{c} \frac{x}{1 !}+\frac{a(a+1) b(b+1)}{c(c+1)} \frac{x^{2}}{2 !} \\
& +\frac{a(a+1)(a+2) b(b+1)(b+2)}{c(c+1)(c+2)} \frac{x^{3}}{3 !}+\cdots .
\end{aligned}
$$

We find the eigenvalue by a shooting method. Given $r^{*}, \lambda^{*}$ is the first positive root of the function

$$
\lambda \mapsto{ }_{2} \mathrm{~F}_{1}\left(\frac{1-\sqrt{1+4 \lambda}}{2}, \frac{1+\sqrt{1+4 \lambda}}{2} ; \alpha+1 ; \frac{1-\cos r^{*}}{2}\right) .
$$

Consider the example of the geodesic triangle $\mathcal{T} \subset \mathbb{S}^{2}$ which is a face of the regular tetrahedral tessellation, whose vertices in the unit sphere could be taken as $\left(\frac{1}{\sqrt{3}}, \pm \sqrt{\frac{2}{3}}, 0\right)$ and $\left(-\frac{1}{\sqrt{3}}, 0, \pm \sqrt{\frac{2}{3}}\right)$. The distance between vertices is $\varepsilon=$ $\cos ^{-1}\left(-\frac{1}{3}\right)$. The diameter, which equals the distance from vertex to center of 
TABLE 1. Domains and eigenvalues. In this table $\delta=$ $\cos ^{-1}(-1 / \sqrt{3})$ and $\varepsilon=\cos ^{-1}(-1 / 3)$. Values not described are taken from $[\mathrm{RT}]$.

\begin{tabular}{|c|llll|}
\hline$G$ & $\mathcal{I}(G)$ & $r^{*}$ & $\lambda_{1}(G)$ & $\lambda_{1}\left(\mathcal{S}\left(r^{*}\right)\right)$ \\
\hline \hline $\mathcal{W}$ & $\infty$ & $\pi$ & $(\alpha+1) \alpha$ & $(\alpha+1) \alpha$ \\
$\mathcal{S}\left(\frac{\pi}{2}\right)$ & $\frac{\pi}{2 \alpha} Z\left(\frac{\pi}{2}\right)$ & $\frac{\pi}{2}$ & $(\alpha+1)(\alpha+2)$ & $(\alpha+1)(\alpha+2)$ \\
$\mathcal{S}(r)$ & $\frac{\pi}{2 \alpha} Z(r)$ & $r$ & $\lambda^{*}$ & $\lambda^{*}$ \\
\hline $\mathcal{W}, \alpha=\frac{3}{2}$ & $\infty$ & 3.14159265 & 3.75 & 3.75 \\
$\mathcal{S}(\delta), \alpha=\frac{3}{2}$ & 2.07876577 & 2.18627604 & 5.00463538 & 5.00463538 \\
$\mathcal{S}(\varepsilon), \alpha=\frac{3}{2}$ & 0.90871989 & 1.91063324 & 6.19561775 & 6.19561775 \\
$\mathcal{S}\left(\frac{\pi}{2}\right), \alpha=\frac{3}{2}$ & 0.30118555 & 1.57079633 & 8.75 & 8.75 \\
$\mathcal{T}$ & 1.88896324 & 2.15399460 & $5.1590 \ldots$ & 5.11641465 \\
$\hat{\mathcal{T}}$ & 1.90831355 & 2.15742981 & $?$ & 5.10421518 \\
\hline
\end{tabular}

the opposite edge, is $\delta=\cos ^{-1}\left(-\frac{1}{\sqrt{3}}\right)$. $\mathcal{T}$ fits inside a wedge sharing a vertex of angle $\frac{2 \pi}{3}$. Writing

$$
\mathcal{T}=\left\{(\rho, \theta): 0 \leq \theta \leq \frac{2 \pi}{3}, \quad 0 \leq \rho \leq r(\theta)\right\},
$$

we find

$$
r(\theta)=\frac{\pi}{2}+\arctan \left(\frac{\cos \left(\theta-\frac{\pi}{3}\right)}{\sqrt{2}}\right) .
$$

At the vertex we have $\alpha=\frac{3}{2}$ so that

$$
Z(r)=\int_{0}^{r} \tan ^{3}\left(\frac{\rho}{2}\right) \sin \rho d \rho=4 \tan \left(\frac{r}{2}\right)+\sin r-3 r .
$$

$\lambda_{1}(\mathcal{T})$ was found numerically in $[\mathrm{RT}$. Using the computer algebra system MAPLE, we numerically integrate

$$
\mathcal{I}(\mathcal{T})=\int_{0}^{\pi / \alpha} Z(r(\theta)) \sin ^{2}(\alpha \theta) d \theta
$$

and solve $\frac{\pi}{2 \alpha} Z\left(r^{*}\right)=\mathcal{I}(\mathcal{T})$ for $r^{*}$ and (23) for $\lambda^{*}$ to get the other values in the $\mathcal{T}$ line in Table 1 .

To avoid the quadrature, we observe the estimate

$$
Z(r(\theta)) \leq T(\theta):=A_{1}+A_{2} \cos \left(\theta-\frac{\pi}{3}\right)+A_{3}(1-\cos (6 \theta)),
$$


where $A_{1}$ and $A_{2}$ are chosen so that the functions agree at $\theta=0$ and $\theta=\frac{\pi}{3}$ and the $A_{3}$ is chosen to make the second derivatives agree at $\frac{\pi}{3}$. The inequality follows since the second derivative of the difference goes from negative to positive in $0<\theta<\pi / 3$. This corresponds to the larger domain $\hat{\mathcal{T}}$ whose radius function is $\hat{r}(\theta)=Z^{-1}(T(\theta))$. Then

$$
\frac{\pi}{2 \alpha} Z\left(\hat{r}^{*}\right)=\int_{\hat{\mathcal{T}}} w^{2} d a=\int_{0}^{\frac{2 \pi}{3}} T(\theta) \sin ^{2}\left(\frac{3}{2} \theta\right) d \theta=\frac{\pi}{3} A_{1}+\frac{9 \sqrt{3}}{16} A_{2}+\frac{\pi}{3} A_{3} .
$$

Using these values we obtain the last row of Table 1. By eigenvalue monotonicity, if $\hat{\mathcal{T}} \supset \mathcal{T}$, then $\lambda_{1}(\mathcal{T}) \geq \lambda_{1}(\hat{\mathcal{T}})$.

This eigenvalue estimate provides an alternative to our argument [RT] in a Brownian pursuit problem. We finished the missing $(n=4)$ case in a proof by $\mathrm{Li}$ and Shao [LS] of the conjecture of Bramson and Griffeath [BG].

Corollary 4. Suppose the prey $X_{0}(t)$ is chased by $n$ pursuers $X_{1}(t), \ldots, X_{n}(t)$, all doing independent standard Brownian motions on the line. Suppose that the pursuers start to the left of the prey $X_{j}(0)<X_{0}(0)$ for all $j=1, \ldots, n$. Then the expected capture time is finite if and only if $n \geq 4$.

In fact, for the capture time for $n$ pursuers,

$$
\tau_{n}=\inf \left\{t>0: X_{j}(t) \geq X_{0}(t) \text { for some } j \geq 1\right\}
$$

there are finite constants $a(n)$ and $C$ depending on the initial position and the eigenvalue of the link of the pursuit cone $[\mathrm{DB}]$ so that the probability

$$
\mathbb{P}\left(\tau_{n}>t\right) \sim C t^{-a} \quad \text { as } t \rightarrow \infty .
$$

The proof shows $a(n)>1$ and thus $\mathbb{E} \tau_{n}<\infty$ if and only if $n \geq 4$. Our eigenvalue estimates give the following corresponding bounds on the decay rates since they are related by a formula to the eigenvalue estimates [RT]. From the estimate on $\hat{\mathcal{T}}, a(3) \geq .90695886$ and so $a(4) \geq 1.00029446$; from the estimate of $\mathcal{T}$ involving quadrature, $a(3) \geq .90827616$ and $a(4) \geq 1.00151234$.

Proof. Details are provided in [RT]. Finiteness of the expectation of $\tau_{4}$ follows if it can be shown that $\lambda_{1}(\mathcal{T})>5.101267527$. The lower eigenvalue bound is given by Theorem 1 applied to $\mathcal{T}$ and depends on either the numerical integration of $\mathcal{I}(\mathcal{T})$ or its upper bound by the quadrature free estimate of (24).

\section{ACKNOWLEDGEMENTS}

The second author thanks the University of California, Irvine, for his visit while this work was being completed. We also thank Lofti Hermi for bringing [PW] to our attention.

\section{REFERENCES}

[BG] M. Bramson and D. Griffeath, Capture problems for coupled random walks. In Random Walks, Brownian Motion and Interacting Particle Systems (R. Durrett and H. Kesten, eds.). Birkhäuser Boston, 1991. MR1146445 (93e:60192)

[Ch] I. Chavel, Eigenvalues in Riemannian Geometry. In Pure and Applied Mathematics, 115. Academic Press, Inc., Orlando, FL, 1984. MR768584 (86g:58140)

[C] J. Cheeger, A lower bound for the smallest eigenvalue of the Laplacian. In Problems in Analysis. Princeton University Press, 1970. MR0402831 (53:6645)

[DB] R. D. DeBlassie, Exit times from cones in $\mathbb{R}^{n}$ of Brownian motion. Prob. Theory and Rel. Fields 74 (1987), 1-29. MR863716 (88d:60205) 
[F] C. Faber, Beweiss, dass unter allen homogenen Membrane von gleicher Fläche und gleicher Spannung die kreisförmige die tiefsten Grundton gibt. Sitzungsber.-Bayer. Akad. Wiss., Math.-Phys. Munich (1923), 169-172.

[K] E. Krahn, Über eine von Rayleigh formulierte Minimaleigenschaft des Kreises. Math. Ann. 94 (1925), 97-100. MR 1512244

[LT] P. Li and A. Treibergs, Applications of eigenvalue techniques to geometry. Contemporary Geometry: J.-Q. Zhong Memorial Volume (H.-H. Wu, ed.). University Series in Mathematics, Plenum Press, New York, 1991, pp. 22-54. MR1170358 (93i:58159)

[LS] W. Li and Q.-M. Shao, Capture time of Brownian pursuits. Prob. Theory and Rel. Fields 121 (2001), 30-48. MR:1857107 (2002h:60173)

[P] L. Payne, Isoperimetric inequalities and their applications. SIAM Review 9 (1967), 453-488. MR 0218975 (36:2058)

[PW] L. Payne and H. Weinberger, A Faber-Krahn inequality for wedge-like membranes. Journal of Mathematics and Physics 39 (1960), 182-188. MR0128158(23:B1202)

[RT] J. Ratzkin and A. Treibergs, A capture problem in Brownian motion and eigenvalues of spherical domains. Trans. Amer. Math. Soc., 361 (2009), 391-405.

[Sz] G. Szegö, Über eine Verallgemeinerung des Dirichletschen Integrals. Math. Zeit. 52 (1950), 676-685. MR0040493(12:703c)

Department of Mathematics, University of Georgia, Athens, Georgia 30602

Current address: School of Mathematical Sciences, Aras Na Laoi, University College Cork, Cork, Ireland

E-mail address: J.Ratzkin@ucc.ie

Department of Mathematics, University of Utah, Salt Lake City, Utah 84112

E-mail address: treiberg@math.utah.edu 